\title{
Appropriateness Use Criteria of Trans-Thoracic 2D-Echocardiography Among Adult Patients in the Philippine General Hospital
}

\author{
Danielle Louis Villanueva*, Marion Patricio, Jose Donato Magno, Felix Eduardo Punzalan \\ Department of Medicine, University of the Philippines - Philippine General Hospital, Manila, Philippines \\ Email address: \\ louievillanuevamd@gmail.com (D. L. Villanueva) \\ ${ }^{*}$ Corresponding author \\ To cite this article: \\ Danielle Louis Villanueva, Marion Patricio, Jose Donato Magno, Felix Eduardo Punzalan. Appropriateness Use Criteria of Trans-Thoracic \\ 2D-Echocardiography Among Adult Patients in the Philippine General Hospital. Cardiology and Cardiovascular Research. \\ Vol. 4, No. 2, 2020, pp. 27-33. doi: 10.11648/j.ccr.20200402.11
}

Received: March 6, 2020; Accepted: March 23, 2020; Published: April 14, 2020

\begin{abstract}
Background: There is an increasing utilization rate and clinical importance of performing echocardiography especially in a tertiary referral center such as the UP-PGH. An appropriate use criteria (AUC) in echocardiography is essential to improve clinical outcomes while preserving hospital and patient resources. Methods: We reviewed the echocardiographic requests of patients referred for transthoracic 2d-echocardiography. Echocardiography indication were documented mainly using the echocardiography order/request form. The echocardiography indications were adjudicated by at least two investigators. If there was more than one indication that fit into the AUC 2011, the most appropriate one was retained. Results: A total of 1006 echocardiographic requests were included in this study. The patients were predominantly males $(68.2 \%)$ and with a mean age of $56.27 \pm 16.89$. majority of the referrals came from the medicine wards $(37.3 \%)$. The top three cardiac diagnosis are: Heart Failure (25.9\%), Acute Coronary Syndrome (20.7\%) and Hypertension (15.5\%). Of the top 15 most common indications, 11 were appropriate while 2 were maybe appropriate, and 2 were rarely appropriate. The three most common specific indication is the evaluation for heart failure $(21.4 \%)$, the search for a cardiac etiology for a particular symptom $(14.3 \%)$ and the initial evaluation of a suspected hypertensive heart disease $(9.9 \%)$. The most common rarely appropriate indication is the routine perioperative evaluation of ventricular function (5.5\%). Majority of the echocardiography requests have appropriate indications $(87.9 \%)$. Conclusion: This is the first application of the AUC to the transthoracic 2d-echo here in the Philippines. The transthoracic 2Dechocardiography done in the Philippine General Hospital have mostly appropriate indications.
\end{abstract}

Keywords: AUC, Appropriateness Use Criteria, Transthoracic 2d-echo, Philippine General Hospital, Philippine Echocardiography

\section{Introduction}

Transthoracic echocardiography (TTE) is one of the mostcommonly used tests in clinical cardiology. It is a widely available, cost effective, and non-invasive test that can provide substantial and valuable data regarding initial diagnosis, management decisions, evaluation of cardiac status, and clinical follow-up of patients. Moreover, echocardiography may be performed on an elective basis or in an emergency setting.

The University of the Philippines - Philippine General Hospital (UP-PGH) is the national university hospital and the largest tertiary care medical center with a 1500-bed capacity, providing diverse and subspecialized medical and surgical services, both in the inpatient and outpatient settings. Being a national university hospital and referral center, the services of PGH are mainly directed to the underserved strata of the population. In 2013, the hospital attended to almost 600 hundred thousand service patients. Of these, almost $98 \%$ are classified as indigent or those heavily relying on the hospital's resources for their medical care. [1] Hence, there is a need to rationalize the resources and redirect them to those patients who truly need them.

The Division of Cardiovascular Medicine of the 
Department of Medicine operates the adult echocardiography laboratory of the hospital. The lab provides ultrasound-based examinations such as the transthoracic echocardiography (TTE), trans-esophageal echocardiography (TEE), stress and contrast echocardiography and vascular duplex studies. These ultrasound-based examination procedures comprised $89 \%$ of all diagnostic procedures done in the year 2016 . The resting transthoracic 2d-echo comprised $94 \%$ of the diagnostics done at the PGH Section of Cardiology echocardiography lab in 2016. [2] There has been an average of $5.8 \%$ increase in the utilization of TTE per year since 2014. In the year 2017, the echocardiography lab accommodated 6,295 patients, which is $6.4 \%$ higher than last year. [2]

In light of its multiple and diverse uses, the appropriate use criteria (AUC) in echocardiography are essential to improve clinical outcomes while preserving hospital and patient resources. Clinically, the application of AUC avoids potential over-indications; from a socio-economic perspective, AUC prevents unnecessary health expenses in institutions with a relatively restricted health care budget such. [3]

The latest AUC used for echocardiography was jointly published in 2011 by the American College of Cardiology Foundation, the Appropriate Use Criteria Task Force, and the American Society of Echocardiography (AUC 2011). [4] The AUC 2011 consisted of 98 indications for transthoracic 2decho. Each indication was designated as appropriate use (median 7-9), uncertain use (median 4-6), and inappropriate use (median 1-3). [4] The PGH Section of Cardiology also released its own Clinical Echocardiography Appropriate Use Criteria for TTE based mainly on AUC 2011 [5]. Apart from these guidelines, there seem to be no other published studies focusing on the clinical value of appropriate use criteria in transthoracic $2 \mathrm{~d}$-echocardiography at the local front. Through this study, we intend to evaluate the appropriateness of transthoracic echocardiograms in a high-volume tertiary medical center using established appropriate use criteria.

The UP-PGH, as the premier tertiary referral hospital, aims to render quality health care by serving as a role model in healthcare delivery systems and leading the medical community in undertaking health systems researches. The resting transthoracic $2 \mathrm{~d}$-echo comprises $94 \%$ of the diagnostic procedures performed at the PGH Section of Cardiology echocardiography lab [4]. In international studies on cardiac imaging utilization, with the first implementation of the AUC in 2007, there was a decline in the volume of cardiac imaging yearly from 2009 to 2013 [6]. The application of the AUC 2011 among hospitals in the US, UK and Australia have shown that their echocardiographic requests are $80-90 \%$ appropriate and that $1-2$ out of 10 requests can be avoided. [7-10] The correction in the utilization of echocardiographic procedures decongested the volume of imaging in the lab and improved the utilization of hospital resources [6-10]. This study, however, is not intended to decrease the volume of procedures at the lab. Historical records of the hospital clearly show a steady rise in the demand for echo. This, in fact, will be further augmented by creating a greater awareness of clear indications for echocardiography, allowing the laboratory to serve a greater segment of the population. More importantly, the AUC will refine cardiac imaging by redirecting its use towards scenarios where it may facilitate cost effective optimal care.

Comparison between the appropriateness of echocardiography between regional and national hospitals are not that different. In a study by Bailey et al., [11] they found that the adherence to the AUC 2011 regarding inpatients in a regional hospital was consistent with that encountered in university hospitals. The application also of the AUC in the community setting was fairly comparable in an Italian study in 2012 by Ballo et al., the adherence in the AUC 2011 in a community setting was only appropriate for $80 \%$ of the studies. [12]

In addition, the results of this study may be used for the improvement of the healthcare delivery system of the hospital specifically in terms of cardiac diagnostics. It may help create a quality improvement project (QIP) regarding TTE indications which includes regular lectures for medical staff on AUC 2011, placement of visual aids summarizing AUC 2011 in the echo lab, and performance of monthly auditing by cardiology fellows in the echo lab.

\section{Research Question}

How appropriate is the indication to perform a transthoracic 2D-echocardiography as assessed by the appropriateness use criteria?

\section{Objectives}

General Objective

To determine the appropriateness of indication to perform a transthoracic 2D-echocardiography in UP-PGH using the appropriateness use criteria.

Specific Objectives

1. To describe the demographics of patients undergoing a transthoracic 2D- echocardiography of the study population.

2. To determine the clinical indication for performing a transthoracic 2D- echocardiography of the study population.

3. To determine the appropriateness of indication using the appropriateness use criteria (ASE 2011 and Multimodality Criteria 2019).

\section{Methodology}

Setting: The study was conducted in the Division of Cardiovascular Medicine - Echocardiography Lab of the Philippine General Hospital. The Section and the echo lab is located at the 6th Floor of the UP-PGH Central Block Building. Study Population

All requests and examinations of adult patients admitted in UP-PGH for resting two-dimensional and Doppler echocardiographic study was included in the study.

Inclusion Criteria

All elective two-dimensional and Doppler echocardiographic request and examination of adult patients 
$\geq 19$ years old admitted in UP-PGH under the service ward was included in the study.

Exclusion Criteria

Two-dimensional echocardiography requests with additional studies aside from Doppler study, such as stress echocardiogram and contrast echocardiogram. Requests for Trans-esophageal echocardiogram (TEE) were also excluded.

Study Design

This is an observational study design that determined the appropriateness of indication to perform a transthoracic 2Dechocardiography in UP-PGH using the appropriateness use criteria.

Overview of Methods

Requests for elective 2D-echocardiography were screened for this study. Data gathered included the age, sex, source of referral, cardiac diagnosis, and echocardiographic indication. The cardiac diagnosis and echocardiographic indication was documented mainly using the echocardiography order/request form and the patient's medical records. The indication was labeled according to appropriateness use criteria scores (AUC) as appropriate (score 7 - 9), maybe appropriate (score 4 - 6) and rarely appropriate (score 1 - 3). If the echo indication in the echo order/request form or the patient's medical records is unclear or not stated, then the AUC is labelled was unknown. The echocardiography indications were adjudicated by the two investigators.

The different sources of referral are the wards, the critical care units, and the emergency rooms. The wards include the medical wards (Ward 1 and 3), surgical wards (ward 2, 4, 6, 8,10 ), neurosciences ward (ward 5), obstetrics ward (ward 15 and 16) and gynecology ward (ward 14b). The critical care units include the medical intensive care unit (MICU), surgical intensive care unit (SICU), the post-anesthesia care unit (PACU), and intensive maternal unit (IMU). The emergency rooms include the acute care unit (ACU) which is the main unit that receives new emergency referrals, the $\mathrm{OB}$ admitting section (OBAS) which is the emergency room for obstetric patients, and the labor room/delivery room (LRDR) which caters to pregnant patients for imminent delivery.

Study Duration: 6 months (January to June 2019).

Sample Size

Convenience sampling method was employed wherein any request for resting TTE studies of adult patients was included as long as they fulfilled the inclusion criteria.

\section{Data Management and Analysis}

Data analysis will be performed using the software Statistical Package for the Social Sciences. Data will be expressed as mean \pm standard deviation, or number and percentage, as appropriate.

\section{Ethical Considerations}

No identifiers were recorded and data collected remained confidential. There were no direct risks or benefits of the study to the participants. There were no form of incentive or compensation given. The study protocol adhered to the Data Privacy Act of 2012 and was approved by the University of the Philippines Manila Research Ethics Board (UPMREB).

\section{Results}

Baseline Demographics and Source of Referral.

A total of 1006 echocardiographic requests were included in the study. Table 1 lists the demographics and source of referral of the patients. The patients were predominantly males $(68.2 \%)$ and with a mean age of $56.27 \pm 16.89$. Of the 1006 included patients, $52.4 \%$ were referred from the medicine areas. Among these, majority $(37.7 \%)$ were from medicine ward 1 and 3 . Among patients referred from non-medicine areas, most of the referrals were from the Surgery ward (17.1\%), Obstetrics and Gynecology ward (7.7\%), and Neurosciences ward (4.6\%). While most of the requests from the critical care units were from the medical ICU (14.7\%), the acute care unit (4.9\%), the surgical ICU (4.6\%), the post anesthesia care unit $(3.3 \%)$, and the intensive maternal unit $(2.0 \%)$.

Table 1. Demographics and Source of Referral of the Study Population.

\begin{tabular}{l|l}
\hline & $\mathbf{N}=\mathbf{1 0 0 6} \mathbf{\%}$ \\
\hline $\begin{array}{l}\text { Age (mean) } \\
\text { Gender }(\mathrm{N}, \%)\end{array}$ & $56.27 \pm 16.89$ \\
Male & $688(68.38 \%)$ \\
Female & $318(31.6 \%)$ \\
Source of Referral & \\
Medicine & \\
Wards & $377(37.5 \%)$ \\
ICU & $153(15.2 \%)$ \\
Neurosciences & \\
Wards & $46(4.6 \%)$ \\
ICU & $23(2.3 \%)$ \\
Surgery & \\
Ward & $171(16.99 \%)$ \\
ICU & $46(4.6 \%)$ \\
OB-Gynecology & \\
Obstetrics & $58(5.8 \%)$ \\
IMU & $20(2.0 \%)$ \\
Gynecology & $19(1.9 \%)$ \\
Peripheral Areas & \\
Acute Care Unit & $49(4.9 \%)$ \\
PACU & $33(3.3 \%)$ \\
OBAS/LRDR & $11(1.1 \%)$ \\
\hline
\end{tabular}

General Cardiac Diagnosis of the Study Population.

Table 2 lists the different cardiac diagnosis indicated in the echo requests. Heart failure was the most common diagnosis specified in the echo request. The top three diagnosis are: Heart Failure (25.9\%), Acute Coronary Syndrome (20.7\%) and Hypertension (15.5\%). Syncope of possible cardiac origin accounted for the least requested diagnosis.

Table 2. Cardiac Diagnosis of the Study Population.

\begin{tabular}{ll}
\hline Cardiac Diagnosis & $\mathbf{( N , ~ \% )}$ \\
\hline Heart Failure & $259(25.7 \%)$ \\
Acute Coronary Syndrome & $213(21.17 \%)$ \\
Hypertension & $155(15.4 \%)$ \\
Suspected Cardiac Etiology & $81(8.1 \%)$ \\
Pre-Chemotherapy & $57(5.7 \%)$ \\
Pre-operative/Pre-procedure Risk Assessment & $55(5.5 \%)$ \\
\hline
\end{tabular}




\begin{tabular}{ll}
\hline Cardiac Diagnosis & $\mathbf{( N , ~ \% )}$ \\
\hline Cardiac Source of Emboli & $49(4.9 \%)$ \\
Arrhythmias & $27(2.7 \%)$ \\
Valvular Heart Disease & $24(2.4 \%)$ \\
Structural/Congenital Heart Disease & $20(2.0 \%)$ \\
Pericardial Disease & $16(1.6 \%)$ \\
Acute Pulmonary Embolism & $16(1.6 \%)$ \\
Aortic Dilation / Aortic Dissection & $10(1.0 \%)$ \\
Infective Endocarditis & $8(0.8 \%)$ \\
Cardiomyopathy & $6(0.6 \%)$ \\
Pulmonary Hypertension & $4(0.4 \%)$ \\
Prosthetic Valves & $4(0.4 \%)$ \\
Syncope of Possible Cardiac origin & $2(0.2 \%)$ \\
\hline
\end{tabular}

Specific Echocardiographic Indications and the

\section{Appropriateness Use Criteria.}

Among the 98 Appropriateness Use Criteria (AUC) established by the American Society of Echocardiography in 2011 and Multimodality Criteria in 2019, only 26 criteria were identified in this study population. The top fifteen specific echocardiographic indications are listed in Table 3. The three most common specific indication is the evaluation for heart failure $(21.4 \%)$, the search for a cardiac etiology for a particular symptom $(14.3 \%)$ which included dyspnea, chest pain, and palpitations from a possible cardiac cause, and the initial evaluation of a suspected hypertensive heart disease $(9.9 \%)$. The most common rarely appropriate indication is the routine perioperative evaluation of ventricular function $(5.5 \%)$.

Table 3. Top 15 Specific Echocardiographic Indications and the AUC Score*.

\begin{tabular}{|c|c|c|c|}
\hline & Specific Indications & $\mathbf{N}(\%)$ & AUC Score \\
\hline 1 & $\begin{array}{l}\text { Initial evaluation of known or suspected HF (systolic or diastolic) based on symptoms, signs, or abnormal test } \\
\text { results }\end{array}$ & $215(21.4 \%)$ & Appropriate \\
\hline 2 & $\begin{array}{l}\text { Symptoms or conditions potentially related to suspected cardiac etiology including but not limited to chest } \\
\text { pain, shortness of breath, palpitations, TIA, stroke, or peripheral embolic event }\end{array}$ & $144(14.3 \%)$ & Appropriate \\
\hline 3 & Initial evaluation of suspected hypertensive heart disease & $100(9.9 \%)$ & Appropriate \\
\hline 4 & Initial evaluation of ventricular function following ACS & $88(8.7 \%)$ & Appropriate \\
\hline 5 & $\begin{array}{l}\text { Acute chest pain with suspected MI and non-diagnostic ECG when a resting echocardiogram can be } \\
\text { performed during pain }\end{array}$ & $70(6.9 \%)$ & Appropriate \\
\hline 6 & $\begin{array}{l}\text { Evaluation of a patient without chest pain but with other features of an ischemic equivalent or laboratory } \\
\text { markers indicative of ongoing MI }\end{array}$ & $60(5.9 \%)$ & Appropriate \\
\hline 7 & Baseline and serial re-evaluations in a patient undergoing therapy with cardiotoxic agents & $57(5.7 \%)$ & Appropriate \\
\hline 8 & Routine perioperative evaluation of ventricular function with no symptoms or signs of cardiovascular disease & $55(5.5 \%)$ & Rarely Appropriate \\
\hline 9 & Hypotension or hemodynamic instability of uncertain or suspected cardiac etiology & $35(3.5 \%)$ & Appropriate \\
\hline 10 & Re-evaluation of known hypertensive heart disease without a change in clinical status or cardiac exam & $34(3.4 \%)$ & Maybe Appropriate \\
\hline 11 & Sustained or nonsustained atrial fibrillation, SVT, or VT & $26(2.6 \%)$ & Appropriate \\
\hline 12 & Initial evaluation when there is a reasonable suspicion of valvular or structural heart disease & $24(2.4 \%)$ & Appropriate \\
\hline 14 & Suspected pulmonary embolism in order to establish diagnosis & $16(1.6 \%)$ & Rarely Appropriate \\
\hline 15 & Suspected pericardial conditions & $16(1.6 \%)$ & Appropriate \\
\hline
\end{tabular}

*Based on American Society of Echocardiography Appropriateness Use Criteria 2011 and Multimodality Imaging AUC 2019.

Among the top 15 specific echocardiographic indications, 11 were considered appropriate. However, there were four specific indications that had scores less than 9. Two of which were considered maybe appropriate which are the routine evaluation of a known asymptomatic hypertensive heart disease $(3.4 \%)$ and heart failure $(1.6 \%)$. Two indications were deemed rarely appropriate which are routine echocardiography for perioperative evaluation $(5.5 \%)$ and diagnosis of acute pulmonary embolism (1.6\%).

Majority of the specific indications were deemed appropriate (Table 4). Almost $88 \%$ of the requests had AUC scores between 7-9, of which 78\% were most appropriate. There were 67 requests $(6.7 \%)$ which are maybe appropriate. These were mostly hypertensive and heart failure patients who are asymptomatic and without any change in their clinical status. Lastly, around $7.2 \%$ of the requests were classified as rarely appropriate. These were patients who were referred for routine pre-operative evaluation among asymptomatic patients $(5.5 \%)$, the use of transthoracic echo to diagnose acute pulmonary embolism (1.6\%), and for a young patient with an isolated PVC on her electrocardiogram $(0.2 \%)$.

Table 4. Summary of distribution of Appropriate, Maybe Appropriate, and Rarely Appropriate Indication Rates ( $n=1006)$.

\begin{tabular}{|c|c|c|c|c|}
\hline & Score 9 & Score 8 & Score 7 & Score 7-9 \\
\hline & $\mathrm{N}, \%$ & $\mathrm{~N}, \%$ & $\mathrm{~N}, \%$ & Total N, \% \\
\hline \multirow[t]{3}{*}{ Appropriate } & $782(77.7 \%)$ & $100(9.9 \%)$ & $2(0.2 \%)$ & $884(87.9 \%)$ \\
\hline & Score 6 & Score 5 & Score 4 & Score 4-6 \\
\hline & $\mathrm{N}, \%$ & $\mathrm{~N}, \%$ & $\mathrm{~N}, \%$ & Total N, \% \\
\hline \multirow[t]{3}{*}{ Maybe Appropriate } & $16(1.6 \%)$ & - & $34(3.4 \%)$ & $50(5.0 \%)$ \\
\hline & Score 1 & Score 2 & Score 3 & Score 1-3 \\
\hline & $\mathrm{N}, \%$ & $\mathrm{~N}, \%$ & $\mathrm{~N}, \%$ & Total N, \% \\
\hline Rarely Appropriate & - & $72(7.2 \%)$ & - & $72(7.2 \%)$ \\
\hline
\end{tabular}




\section{Discussion}

As the utility of transthoracic echocardiography (TTE) continues to increase, the health care system needs to understand how to best and appropriately incorporate this technology into daily clinical care. In this study, we provided an estimate of how appropriate the TTE indications in a university hospital is according to the American Society of Echocardiography's AUC 2011 and Multimodality Imaging Criteria 2019. There is no published study on the application of the appropriateness use criteria here in the Philippines. The location is of note because this study was conducted outside the USA, where the AUC 2011 were established. To our knowledge, this is the first study on the AUC application in a Philippine hospital.

Theoretically, the applicability of the AUC in transthoracic echo is expected to be similar for different countries. But this is not the case especially for developing countries where the major difference is in the allocation of the healthcare budget. The AUC 2011 was created and tested in the United States of America. [4] In the United Kingdom, Gurzun and Ionescu [7] reported that the assessment of AUC in the UK showed similar reports to those reported in the USA [8] where 1 out of 10 TTE indications could be avoided. This was also evident in other European centers in a study by Kerley et al. [9] in Ireland where $85 \%$ of studies were appropriate and around $10 \%$ of the studies could have been avoided.

In our study, we have included 1006 echocardiographic requests, of which almost $88 \%$ were deemed appropriate where about 1 out of 10 studies could be avoided. This is almost similar to the results of the studies done in the US and the UK. Interestingly, our results showed more appropriate use of the technology than in a regional center in Australia where only $77 \%$ were appropriate and $20 \%$ were considered inappropriate. [10].

In this study, we found that the most common appropriate indications are the "initial evaluation of known or suspected HF (systolic or diastolic) based on symptoms, signs, or abnormal test results" and "initial evaluation of a suspected hypertensive heart disease". This is an expected and logical finding since almost $50 \%$ of our inpatients in the wards or ICU are admitted with a diagnosis of heart failure as well as hypertension and most of these patients do not have an echocardiogram done in the out-patient clinics. [13] In contrast to the results of published studies on AUC, where the indication "Symptoms or conditions potentially related to suspected cardiac etiology including but not limited to chest pain, shortness of breath, palpitations, TIA, stroke, or peripheral embolic event" is the most common, in our study, it is only the second most commonly requested indication. A possible explanation to this is that most of our inpatients are diagnosed clinically with heart failure and hypertension in rural health centers and small clinics before they come in to our hospital.

Interestingly 4 out of the top 15 requested specific echo indications were deemed not appropriate. The most common least appropriate indication was the "routine perioperative evaluation of ventricular function with no signs or symptoms of cardiovascular disease". We have observed that among those referred for $2 \mathrm{~d}$-echo, most are beyond 60 years old and with stable comorbidities such as hypertension and type 2 diabetes mellitus. We also observed that routine evaluation for known hypertensive and heart failure patients without any change in clinical status are frequently requested for an echocardiogram. We estimate that such routine evaluations do not affect the operative course and may lead to unnecessary health-care expenses; moreover, a suitable history taking with physical examination, along with chest X-rays and electrocardiogram is usually sufficient for evaluation. These findings were similar to a study in 2013 by Matulevicius et al. [13] where they observed that only $31.8 \%$ of echocardiography done led to an active change in the management of the patient while the remaining either led to continuation of care or to no change in the management at all. In this perspective also we highlight on the importance that the request for an echo be assessed by a cardiologist especially when the indication is uncertain. We would also like to improve the dissemination of information regarding the appropriateness indication to request for a transthoracic $2 \mathrm{~d}$-echo among non-cardiologists in our institutions by posting visual materials containing the AUC indications in our echocardiography lab as well as the service areas where these requests are made. In a study by Bhatia et al. [14], AUC based educational intervention on requesting transthoracic $2 \mathrm{~d}$-echo significantly reduced the rate of inappropriate requests and produced a significant reduction in daily echocardiography requests. Such practice would not only allow the preservation of financial resources but also it will allow other patients with more appropriate indications for early access thereby reducing the waiting time in the echo lab.

Though it seems inappropriate based on the AUC 2011, the request for a $2 \mathrm{~d}$-echo for the diagnosis of acute pulmonary embolism is commonly done in our hospital. Most of the patients referred were hypotensive patients with unexplained dyspnea and tachycardia. This is understandable since in our institution the availability of more advanced equipment used in the diagnosis of pulmonary embolism is not readily accessible. The transthoracic $2 \mathrm{~d}$-echo is readily available and inferences can be made from the echo findings that can alter the emergent management of the case. The requesting physician must weigh between the clinical indication as well as the echocardiography indication to provide the best management for the patient [15].

As mentioned in the methodology section, our study only considered one appropriate indication. If the echocardiogram request indicated multiple appropriate indications, the researchers selects the most appropriate indication to be recorded. Other observations that we have are that the echocardiographic forms are frequently not filled up 
completely and properly. The echocardiographic diagnosis and indication should be properly written in order to guide the sonographer and echocardiographer on what to look for and help them identify the abnormalities easily.

Since majority of the echo requests here in our hospital are appropriate and AUC scores are comparable to international hospitals, it is important to maintain the application of the AUC criteria in requesting for transthoracic 2d-echocardiography. The resources of a government run hospital is limited and improvement in healthcare systems delivery is of paramount importance. In the upcoming Universal Health Care implementation, the utilization of hospital resources and services should be appropriate.

\section{Summary and Conclusion}

This study determined the appropriateness in performing a transthoracic 2D-echo in a tertiary resource limited hospital. Majority or $87.9 \%$ of the echocardiographic requests were found to be appropriate, $5.0 \%$ were maybe appropriate, and $7.2 \%$ were rarely appropriate. The most common appropriate indication is the evaluation of heart failure, hypertension, and symptoms possibly related to a suspected cardiac etiology. Based on international studies, the Philippine General Hospital has good compliance to requesting appropriate transthoracic 2D-echocardiography.

\section{Ethical Compliance with Human/Animal Study}

There are no humans or animals included in this study.

\section{Informed Consent}

No informed consent was needed for this study since there were no involvement of any patient or participant.

\section{Authors Contribution}

All authors have directly participated in the planning, and analysis of this study and have read and approved the final version submitted.

\section{Institutional Review Board}

This paper has been registered with the Research and Grants Office of the University of the Philippines - Research Ethical Board. The hospital affiliation: Division of Cardiovascular Medicine, Department of Medicine, University of the Philippines - Philippine General Hospital is fully aware of this submission.

\section{Data Availability Statement}

The data used to support the findings of this study are included within the article. Additional data or information can be requested by contacting the corresponding author.

\section{Disclosure of Conflict of Interest and Funding Source}

This research study was a recipient of the University of the Philippines - Philippine General Hospital research grant 2019 which is funded by the Expanded Hospital and Research Office of the Philippine General Hospital.

Dr. Felix Eduardo Punzalan and Dr. Jose Donato Magno serves as the consultant advisers to the corresponding author Dr. Danielle Louis Villanueva and co-author Dr. Marion Patricio.

\section{Acknowledgements}

I cannot express enough thanks to my co-authors and consultant mentors for their continued support and guidance in the completion of this research. Also this would not have been easily accomplished without the support of the Division of Cardiovascular Medicine and the Department of Medicine.

\section{References}

[1] Philippine General Hospital Statistics. 2013. PGH Statistics [unpublished].

[2] Division of Cardiovascular Medicine, Department of Medicine, Report of Echo Statistics. 2017. [unpublished].

[3] Amita Singh and R. Parker Ward. 2016. Appropriate Use Criteria for Echocardiography: Evolving Applications in the Era of Value-Based Healthcare. Curr Cardiol Rep. 2016 September; 18 (9): 93.

[4] Douglas P, Garcia, M, Haines, D, et. al. 2011. Appropriate Use Criteria for Echocardiography. J Am Soc Echocardiography, 2011; 24: 229-67.

[5] Magno, Jose Donato. 2018. Clinical Echocardiography Appropriate Use Criteria (The CLEAR Guidelines) for Transthoracic 2-dimensional Echocardiography (2018 Update). [unpublished].

[6] Byrd BF $3^{\text {rd }}$, Abraham TP, Buxton DB, et al. 2015. A Summary of the American Society of echocardiography foundation value based healthcare summit 2014: The role of cardiovascular ultrasound in the new paradigm. J Am Soc Echocardiogr: Off Publ Am Soc Echocardiogr; 28: 755-69.

[7] Gurzun MM and Ionescu A. 2014. Appropriateness of use criteria for transthoracic echocardiography: are they relevant outside the USA. Eur Heart J Cardiovasc Imaging; 15 (4): $450-5$.

[8] Patil HR, Coggins TR, Kusnetzky LL, et al. Evaluation of appropriate use of transthoracic echocardiography in 1,820 consecutive patients using the 2011 revised appropriate use criteria for echocardiography. Am J Cardiol. 2012; 109 (12): 1814-7. 
[9] Kerley RN, Thornton KP, Kelly RM, et al. 2018. Appropriate use criteria for transthoracic echocardiography: Are they relevant to European Centers. Jan; 35 (1): 17-23. Epub 2017 Nov 21.

[10] Al-Kaisey A, Jones E, Nadurata V, et al. 2015. Appropriateness use of echocardiography in an Australian Regional Centre. Intern Med J. 2015; 45 (11): 1128-33).

[11] Bailey SA, Mosteanu I, Tietjen PA, et al. 2012. The use of transthoracic echocardiography and adherence to appropriate use criteria at a regional hospital. J Am Soc Echocardiogr. 2012; 25 (9): 1015-22.

[12] Ballo P, Bandini F, Capecchi I, et al. 2012. Application of 2011 American College of Cardiology Foundation/American Society of Echocardiography appropriateness use criteria in hospitalized patients referred for transthoracic echocardiography in a community setting. $J$ Am Soc Echocardiogry. 2012; 25 (6): 589-98.

[13] Matulevicius SA, Rohatgi A, Das SR, Price AL, DeLuna A, Reimold SC. Appropriate use and clinical impact of transthoracic echocardiography. JAMAIntern Med. 2013; 173 (17): 1600-7.

[14] Bhatia RS, Milford CE, Picard MH, Weiner RB. An educational intervention reduces the rate of inappropriate echocardiograms on an inpatient medical service. JACC Cardiovasc Imaging. 2013; 6 (5): 545-55.

[15] Ioannidis JP. Appropriate vs clinically useful diagnostic tests. JAMA Intern Med. 2013; 173 (17): 1607-9. 